\title{
Clinical Examination of the Water-Soluble V itamin Levels in Blood during Peripheral Parenteral Nutrition
}

\author{
HIROYUKI OZASA, NOBUYA ISHIBA SHI, SATORU IKEDA, TA KUYA IMAIZUMI, \\ MOTOSHI MIYAGI, SHOUJIROU YANO, KEISHIROU AOYAGI, YOSHITO AKAGI, \\ YUTAKA OGATA AND KAZUO SHIROUZU \\ Department of Surgery, Kurume University School of Medicine, \\ Kurume 830-0011, Japan
}

Received 15 September 2006, accepted 5 December 2006

\begin{abstract}
Summary: The water-soluble vitamin (included vitamin B1, B6, B12 and C) preparations are not always replenished when peripheral parenteral nutrition (PPN) is used in Japan. We evaluated the need for adminis tration of vitamins preparation during PPN, and involved analysis of the blood levels of water-soluble vita mins in patients receiving perioperative PPN before and after gastrectomy. Patients were examined as two set of groups as follows; 18 patients who did not receive water-soluble vitamin preparations during PPN, the Unsupplemented Group, and 22 patients who received such preparations during PPN, the Supplemented Group. Consequently, in the Unsupplemented Group, the blood vitamin B1 level during the early postopera tive period was significantly lower than the preoperative level, but in the Supplemented Group, it was significantly higher than the preoperative level. In the Supplemented Group, the blood vitamin B12 level during the early postoperative period was markedly higher than the preoperative level. A nd in both groups, the blood vitamin $\mathrm{C}$ level remained below the lower limit of the criterion range throughout the perioperative period. These results suggested that administration of water-soluble vitamins during PPN was needed to avoid potential vitamin deficiencies after surgery and to prevent a potential onset of severe metabolic complications from any deficiencies.
\end{abstract}

Key words water-soluble vitamin, peripheral parenteral nutrition (PPN), micronutrient

\section{INTRODUCTION}

Recent advances in parenteral and enteral alimenta tion have contributed to improving the nutrition state of patients. For example, total parenteral nutrition (TPN), which was developed in 1967 by Dudrick et al. [1], has been used extensively as a means of nutrition for patients who are unable to receive peroral ingestion or enteral nutrition, or who can do so only with difficulty. However, TPN have resulted in progressively larger number of patients with metabolic complications [2-3], such as vitamin B1 deficiency
(Wernicke encephalopathy, lactic acidosis, etc.) and other complications of deficiencies in micronutrients (vitamins, trace elements, etc.) [4-10]. For this rea son, the guideline prepared by the A merican Society for Parenteral and Enteral Nutrition (ASPEN) have recommended that care should be taken to ensure intake of these elements in the amounts needed daily by patients receiving nutrition with this method [11].

Peripheral parenteral nutrition (PPN) is simpler and cost less than TPN. Because of these features, PPN is often used to provide nutrients. PPN involves administration of lower amounts of carbohydrates

Corresponding address to: Hiroyuki Ozasa, MD., Department of Surgery, Kurume University School of Medicine, 67 A sahi-machi, Kurume $830-0011$, Japan. Tel: +81-942-31-7566 Fax: +81-942-34-0709 E-mail address: hiozasa@ med.kurume-u.ac.jp

A bbreviations: BMI, body mass index; CLEIA, chemiluminescent enzyme immunoassay; DG, distal gastrectomy; GIST, gastrointestinal stromal tumor; HPLC, high performance liquid chromatography; IRB, institutional review board; LAPG, Iaparoscopic-assisted partial gastrectomy; PG, partial gastrectomy; PPG, pylorus preserving gastrectomy; PPN, peripheral parenteral nutrition; SG, segmental gastrectomy; TPN, total parenteral nutrition. 
and its duration is short (about 1-2 weeks) [12], so that deficiency of micronutrients is considered unlikely to occur with this method. A s a result, vita mins are not always prescribed when PPN is used in Japan. A Ithough the guidelines mentioned above recommendation that adequate care should be taken to ensure intake of the required amounts of vitamins every day when parenteral nutrition is used, the optimal dosage and method of administration of micronutrients during PPN are still unclear. Furthermore, although administration of vitamins during the perioperative period is considered preferable because of their antioxidative effects and the involvement of vitamins in glucose and protein metabolism, no detailed criteria are available for dosages or method of administration of vitamins during this period. Moreover, under the current health insurance system in Japan, reimbursement for concomitant use of water-soluble vitamin preparations is limited during PPN. In these circumstances, not a little cases of severe metabolic disease developing during PPN due to vitamin B1 deficiency have been reported [13-14], resembling those previously reported in association with use of TPN. These reports have been suggesting that patients with certain diseases might be suffered potential vitamin deficiency.

The aims of this study were to examine the need for administration of water-soluble vitamin prepara tions during PPN, and involved analysis of the blood levels of water-soluble vitamins (B1, B6, B12 and C) in patients receiving perioperative PPN before and after gastrectomy.

\section{SUBJECTS AND METHODS}

\section{Subjects}

The subjects of this study were patients who were scheduled to receive PPN after gastrectomy, at the Department of Surgery of Kurume University Hospital. During the period from April 2003 to September 2005, 40 patients were enrolled in this study, including 18 patients who received no watersoluble vitamin preparations during PPN-the Unsupplemented Group, and 22 patients who received such preparations during PPN-the Supplemented Group.

Prior to the start of the study, all candidates were informed of the study design, and their consent to participate in the study was obtained in writing. (For candidates incompetent to provide consent, consent was obtained from their guardians.) Institutional review board (IRB)-No.2258 for the Unsupplemented Group was approved at 16th Dec. 2002 by IRB for Kurume University School of Medicine. On the other side, we thought that blood sampling and measurement of blood vitamin levels for the Supplemented Group are one of the common practice act. Therefore, we did not ask for IRB's approval as to these act.

All patients enrolled in this study satisfied all of the following requirements [1] in good general condition and able to ingest food and drink before surgery; [2] with no severe medical complication before surgery; [3] scheduled to receive PPN after surgery; and [4] providing informed consent to participate in the study in writing, accompanied by written consent of their attending physicians.

A dditionally the following exclusion criteria were applied; [1] severe hepatic, renal, or cardiac dy sfunction; [2] severe abnormality in glucose metabolism; [3] hyperkalemia associated with A ddison's disease, severe burns, or azotemia; [4] hyperphosphatemia associated with hypoparathyroidism; [5] hypermagenesemia associated with hypothyroidism; [6] hypercalcemia; and [7] any clear sign of vitamin deficiency.

\section{Experimental protocol (Fig. 1)}

For both the Supplemented and Unsupplemented groups, PPN was performed using multiple electrolyte injection with carbohy drate (electrolyte maintenance solution or extracellular fluid replacement

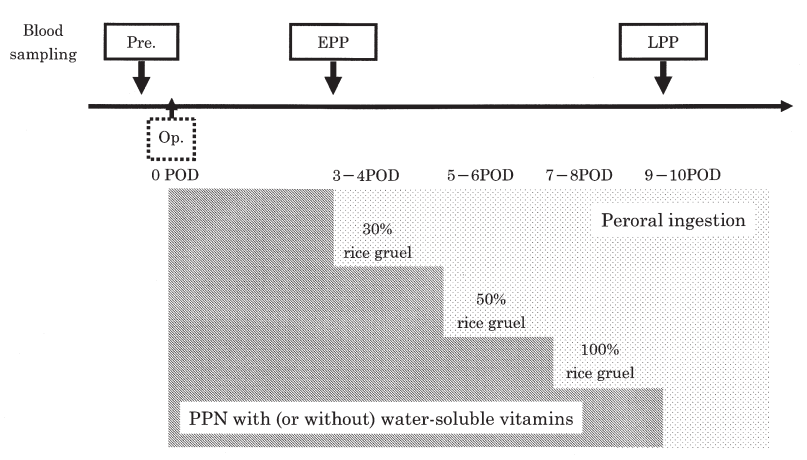

Fig. 1. Experimental protocol. PPN was performed using multiple electrolyte injcetion with carbohydrate (MEIC) or amino acid injection with glucose (AAIG). Administration of the water-soluble vitamin preparation was discontinued when oral ingestion was resumed after surgery. PPN was gradually changed to oral ingestion. For each patient, blood was sampled at 3 time points, i.e., preoperative period ("Pre"), early postoperative period ("EPP”), and late postoperative period ("LPP”). 
solution) or amino acid injection with glucose. After surgery, the volume of IV solution administered was increased or decreased appropriately depending on the condition of each patient, with a target of $30-40$ $\mathrm{ml} / \mathrm{kg} /$ day. The water-soluble vitamin preparations used for the Supplemented Group included vitamin B1 (50 mg/day as thiamine disulfide), vitamin B6 (100 mg/day as pyridoxine hydrochloride), vitamin B12 (1 mg/day as hydroxocobalamin acetate), and vitamin C (500 mg/day). These preparations were used to prescribe to the patients who were underwent gastrectomy in our hospital. The water-soluble vita min preparations were administered together with the initial $500 \mathrm{ml}$ of the IV fluid used for each day over a period of around 5-6 hrs, while covered and shielded from the light. Administration of the water-soluble vitamin preparations were discontinued when peroral ingestion was resumed after surgery. The amount of PPN was gradually reduced and peroral ingestion was started. Peroral ingestion was started at around 3 days after surgery when judged feasible by the attending physician. The diets which usually administered to gastrectomized patients at our hospital were used. Peroral ingestion was started with 30\% rice gruel, followed by $50 \%$ rice gruel, and then by $100 \%$ rice gruel, at intervals of 2 days. A fter peroral ingestion had reached $100 \%$ rice intake, the patient was discharged from the hospital.

For each patient, ten milliliters of blood was sampled at 3 time points, i.e., preoperatively (Pre.), at immediately before the start of peroral ingestion after surgery (early postoperative period; EPP), and at around 6 days after the start of peroral ingestion (late postoperative period; LPP). A t each time point, blood was collected early in the morning before breakfast.

Methods of measurement of water-soluble vitamin levels in blood

Blood vitamin B1, B6, and C levels were mea sured using high-performance liquid chromatography post-column fluorescence method (HPLC). V itamin B12 was measured with chemiluminescent enzyme immunoassay (CLEIA). Both methods have been recommended by the $\mathrm{V}$ itamin Society of Japan [15].

This study is a pilot study not a double-blind study. For comparisons between two groups, ANOVA was used, followed by testing of the significance of differences with Fisher's PLSD, and between the preoperative and postoperative period, Student's t-test was used. In all analyses, findings of $p<0.05$ were considered significant.

Patients for whom a given parameter was above the lower limit of the criterion range were considered that the vitamin sufficiency was "maintained patients", while those for whom the parameter was less than the lower limit of the criterion range were considered "non-maintained patients". The rate of maintenance for each vitamin was calculated using the following equation:

Maintenance rate $(\%)$ = maintained patients/ (maintained patients + non-maintained patients)] $\times$ 100

Fisher's exact method was employed for comparisons of this parameter between the two groups (excluding patients for whom blood sampling had not been performed).

\section{RESULTS}

\section{Background of the patients}

In the Unsupplemented Group, there were 13 males and 5 females, with a mean age of 66.9 2.9 years (mean \pm SEM). The average body mass index (BMI) was 22.8t 0.9. The primary diseases were gastric cancer in 15 cases (Stage I A in 14 cases, and Stage I B in 1 case) [16], and gastric gastrointestinal stromal tumor (GIST) in 3 cases. The operative procedures were distal gastrectomy in 12 cases (reconstruction with Billroth-I procedure in 10 cases, and Roux-en-Y reconstruction in 2 cases), pylorus preserving gastrectomy in 2 cases, partial gastrectomy in 3 cases (open surgery in 1 case, and laparoscopic surgery in 2 cases), and segmental gastrectomy in the other 1 case.

In the Supplemented Group, there were 12 males and 10 females, with a mean age of $66.9 \pm 2.3$ years (meant SEM). The average BMI was 22.24 0.6. The primary diseases were gastric cancer in 21 cases (Stage I A in 15 cases, Stage I B in 3 cases, Stage II in 2 cases, and Stage III A in 1 case) [16], and gastric GIST in 1 case. The operative procedures were distal gastrectomy in 19 cases (reconstruction with Billroth-I procedure in 10 cases, Billroth-II procedure in 2 cases, and Roux-en- $Y$ reconstruction in 7 cases), pylorus preserving gastrectomy in 1 case, open partial gastrectomy in 1 case, and segmental gastrectomy in the other 1 case. In the male to-female ratio, age, primary disease, stage of disease, and operative procedure, there was no significant difference between the two groups.

\section{Parenteral nutrition during fasting period}

The mean postoperative period of fasting was 
$3.5 \pm 0.3$ days in the Unsupplemented Group, 4.1+ 0.1 days in the Supplemented Group. The mean amount of infusion volume and mean daily caloric intake during this period were $2100.5 \pm 85.7 \mathrm{ml}$, $549.7 \pm 30.9 \mathrm{kcal}$ (non-protein calorie intake was $459.7 \pm 24.0 \mathrm{kcal}$ ) in the Unsupplemented Group, $2006.8 \pm 12.4 \mathrm{ml}, 428.6 \pm 19.2 \mathrm{kcal}$ (non-protein calorie intake was $398.6 \pm 9.4 \mathrm{kcal}$ ) in the Supplemented Group, respectively.

\section{Nutrition after resumption of peroral ingestion}

The mean length of time from the start of peroral ingestion to LPP blood sampling was $4.6 \pm 0.3$ days in the U nsupplemented Group, and was $7.2+0.3$ days in the Supplemented Group. From the start of peroral ingestion till LPP blood sampling, the mean daily caloric intake, the mean daily protein intake were $1027.7 \pm 0.4 \mathrm{kcal}, 39.8 \mathrm{~g}$ in the Unsupplemented Group, and were 1031.0 $0.3 \mathrm{kcal}, 39.7 \mathrm{~g}$ in the Supplemented Group. A Iso the mean daily intake of vitamin B1, B6, B12, C were $3.30 \mathrm{mg}, 4.5 \mathrm{mg}, 6.5$ $\mu \mathrm{g}, 277 \mathrm{mg}$ in the Unsupplemented Group, and were
$3.30 \mathrm{mg}, 4.7 \mathrm{mg}, 6.4 \mu \mathrm{g}, 278 \mathrm{mg}$ in the Supplemented Group.

\section{Changes of each blood vitamin levels}

Vitamin B1 (the criterion range; 28 to $56 \mathrm{ng} / \mathrm{ml}$ ) [17]

In the Unsupplemented Group, the blood vitamin B1 level at the EPP $(26.1+2.1 \mathrm{ng} / \mathrm{ml})$ was significantly lower than the preoperative level (30.9 1.6 $\mathrm{ng} / \mathrm{ml}$ ), and did not recover adequately even at the LPP (27.0 $1.5 \mathrm{ng} / \mathrm{ml})$. In the Supplemented Group, the blood vitamin B1 level at the EPP (172.8+ 12.3 $\mathrm{ng} / \mathrm{ml}$ ) was significantly higher than the preoperative

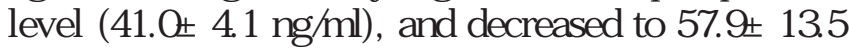
$\mathrm{ng} / \mathrm{ml}$ at the LPP (Fig. 2a).

Vitamin $B 6$ (the criterion range; male: 6 to $40 \mathrm{ng} / \mathrm{ml}$, female: 4 to $19 \mathrm{ng} / \mathrm{ml}$ ) [18]

In the Supplemented Group, the blood vitamin B6 level at the EPP (38.8+ $2.8 \mathrm{ng} / \mathrm{ml}$ ) was significantly higher than the preoperative level $(13.3 \pm 3.9$ $\mathrm{ng} / \mathrm{ml}$ ), and decreased to $16.242 .6 \mathrm{ng} / \mathrm{ml}$ (close to the preoperative level) at the LPP (Fig. 3a).

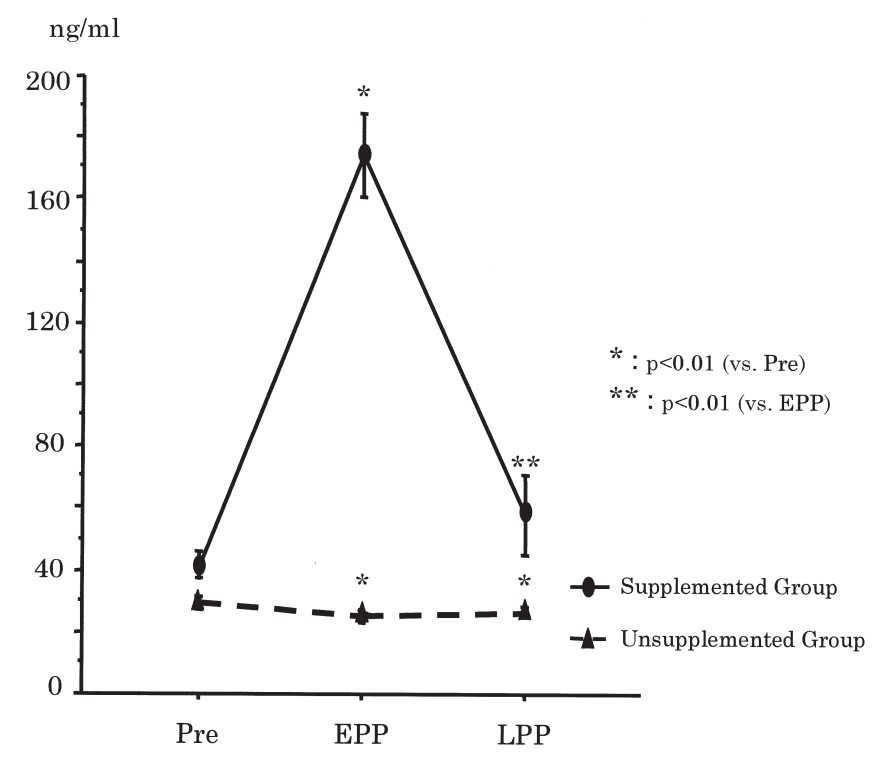

(a)

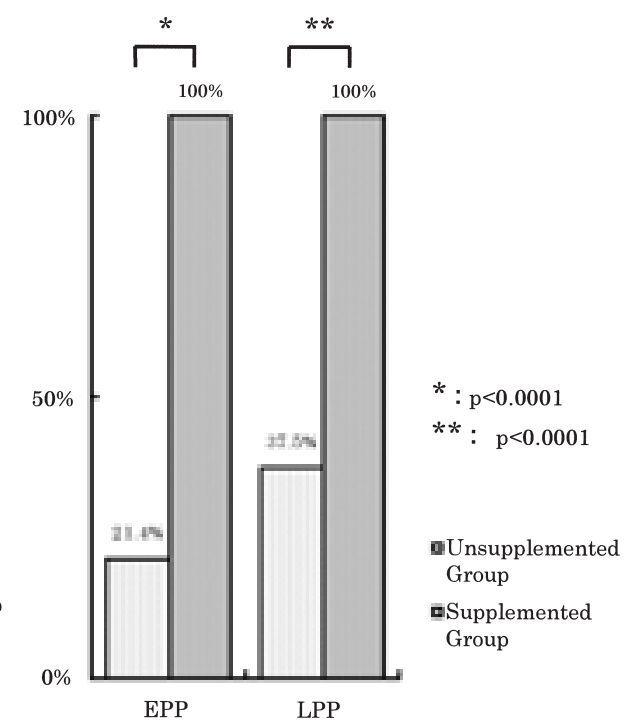

(b)

Fig. 2. Changes and Maintenance rate in the blood vitamin B1 levels. pre: preoperative period, EPP: early postoperative period, LPP: Iate postoperative period

(a) In the Unsupplemented Group, blood vitamin B1 level at the EPP was significantly lower than the preoperative level $(p<0.01)$, and in the Supplemented Group, it was significantly higher than the preoperative level $(p<0.01)$.

(b) The rate of maintenance of blood vitamin B1 at the EPP and LPP was both $100 \%$ in the Supplemented Group, but in the Unsupplemented Group was $21.4 \%$ and $37.5 \%$. 


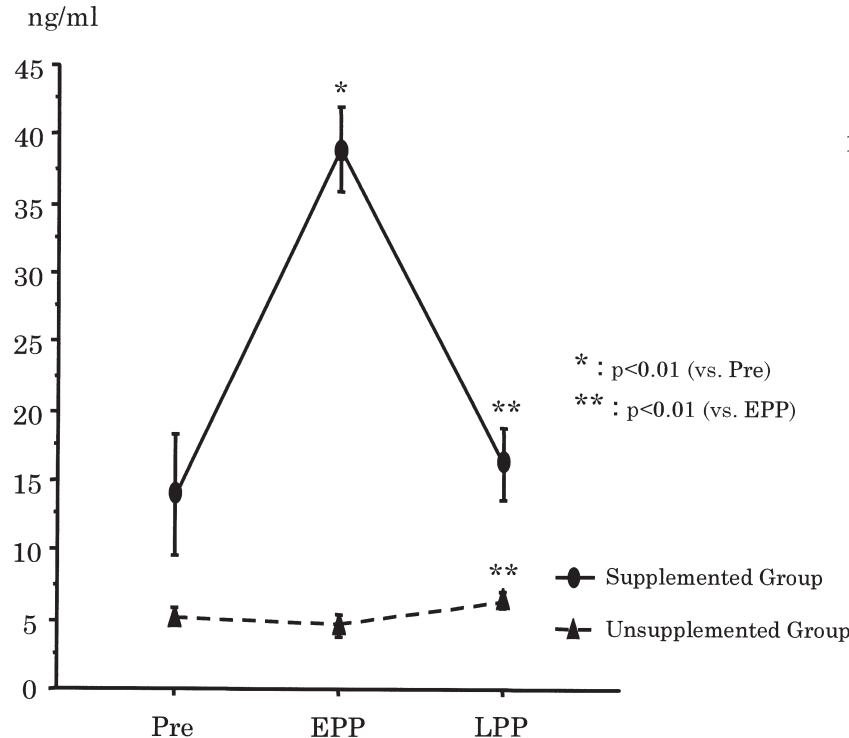

(a)

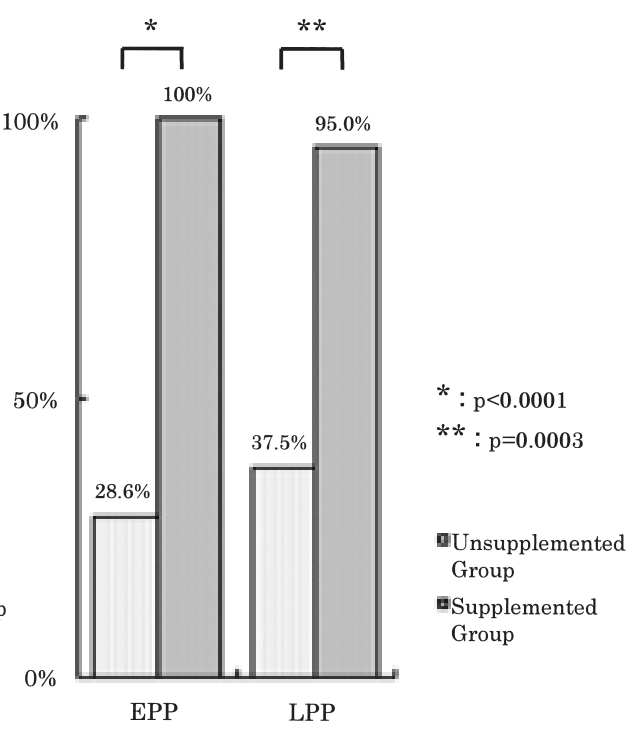

(b)

Fig. 3. Changes and Maintenance rate in the blood vitamin B6 levels. pre: preoperative period, EPP: early postoperative period, LPP: Iate postoperative period

(a) In the Supplemented Group, blood vitamin B6 level at the EPP was significantly higher than the preoperative level $(p<0.01)$.

(b) The rate of maintenance of blood vitamin B6 at the EPP and LPP was both high in the Supplemented Group, but in the Unsupplemented Group was $28.6 \%$ and $37.5 \%$.

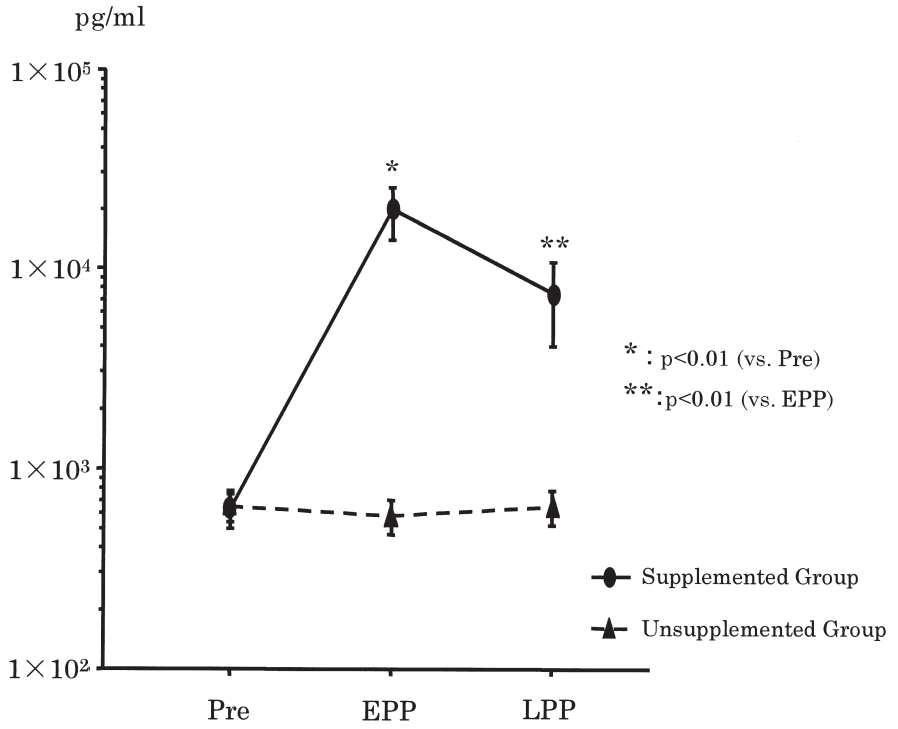

(a)

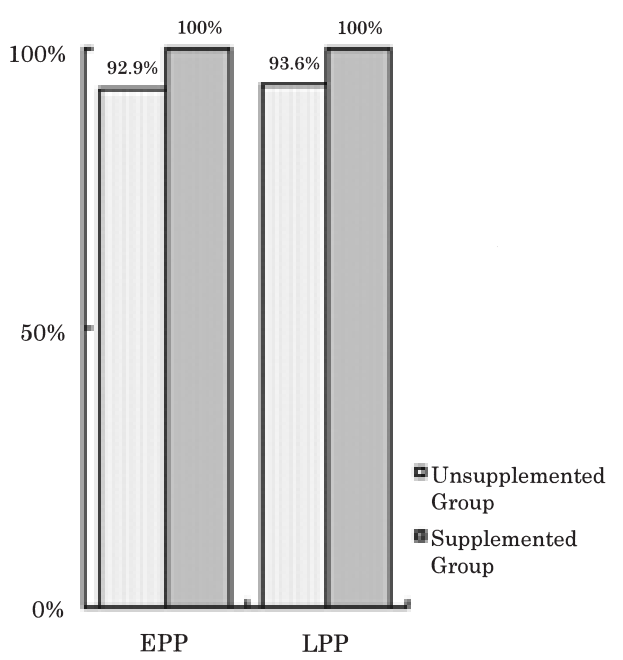

(b)

Fig. 4. Changes and Maintenance rate in the blood vitamin B12 levels. pre: preoperative period, EPP: early postoperative period, LPP: Iate postoperative period

(a) In the Supplemented Group, blood vitamin B12 level at the EPP was markedly higher than the preoperative level $(p<0.01)$, and remained high even at the LPP.

(b) There were no significant differences between the two groups in rate of maintenance of blood vitamin B12 at the EPP or LPP. 


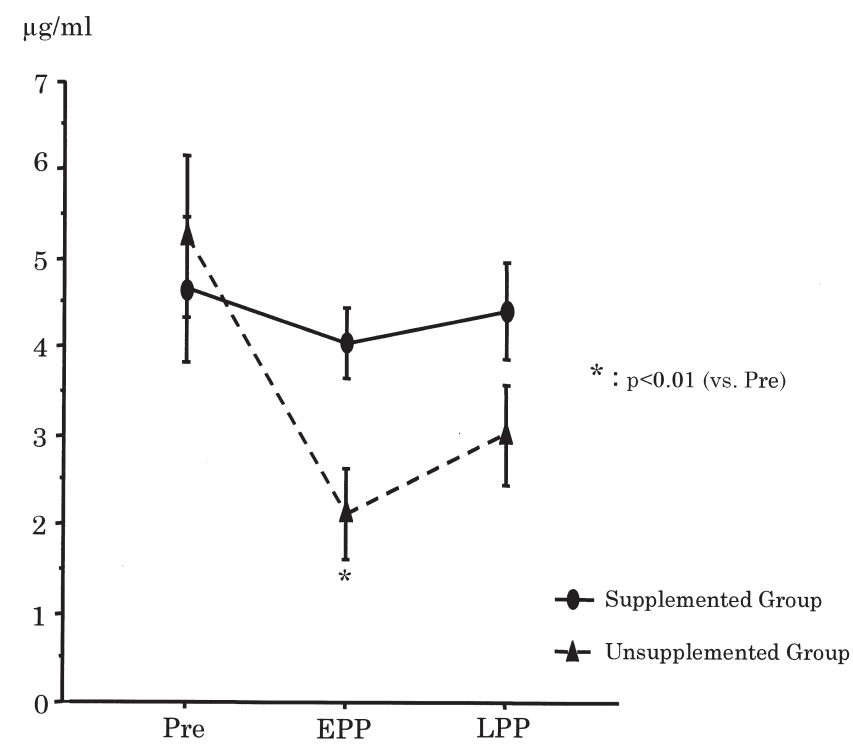

(a)

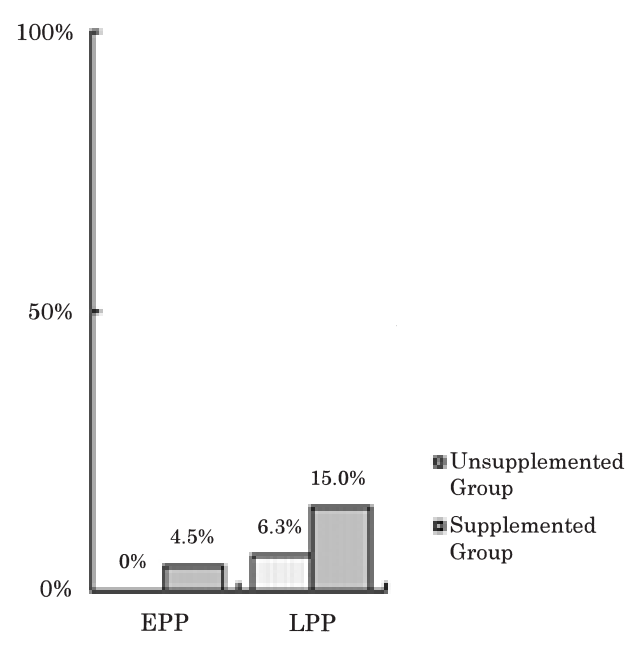

(b)

Fig. 5. Changes and Maintenance rate in the blood vitamin C levels. pre: preoperative period, EPP: early postoperative period, LPP: Iate postoperative period

(a) In the Unsupplemented Group, blood vitamin C level at the EPP was significantly lower than the preoperative level $(p<0.01)$ and did not recover adequately even at the LPP. In both groups, blood vitamin $C$ level remained below the lower limit of the criterion range throughout the perioperative period.

(b) The rate of maintenance of blood vitamin $\mathrm{C}$ did not differ significantly between the two groups at the EPP or LPP, and remained very low in both groups.

Vitamin B12 (the criterion range; 233 to 914 pg/ml) [18]

In the Supplemented Group, the blood vitamin B12 level at the EPP (17781.4 $45310.0 \mathrm{pg} / \mathrm{ml})$ was markedly higher than the preoperative level (607.3+ $114.1 \mathrm{pg} / \mathrm{ml}$ ), and this remained high even at the LPP (7423.5 \pm 3323.5 pg/ml) (Fig. 4a).

Vitamin C (the criterion range; 7.0 to $13.8 \mu \mathrm{g} / \mathrm{ml}$ ) [18]

In the U nsupplemented Group, the blood vitamin C level at the EPP $(2.0 \pm 0.4 \mu \mathrm{g} / \mathrm{ml})$ was significantly lower than the preoperative level $(5.2+0.7 \mu \mathrm{g} / \mathrm{ml})$, and did not increase at the LPP $(3.3 \pm 0.6 \mu \mathrm{g} / \mathrm{ml})$. In the Supplemented Group, the blood vitamin C level remained fairly constant throughout the perioperative period. In both groups, the blood vitamin C level remained below the lower limit of the criterion range throughout the perioperative period (Fig. 5a).
Maintenance rate of each blood vitamin levels (Figs. 2b-5b)

The rate of maintenance of blood vitamin B1 level, B6 level at the EPP were 100\% (22/22), 100\% $(22 / 22)$ in the Supplemented Group, and were $21.4 \%$ (3/14), 28.6\% (4/14) in the Unsupplemented Group, respectively. Both blood vitamin B1 and B6 levels were maintained significantly better in the Supplemented Group than in the Unsupplemented Group at the EPP. At the LPP, the rate of maintenance of blood vitamin B1 level, B6 level were $100 \%(20 / 20), 95 \%(19 / 20)$ in the Supplemented Group, and were $37.5 \%$ (6/16), 37.5\% (6/16) in the Unsupplemented Group, respectively. Thus, both blood vitamin B1 and B6 levels were maintained significantly better in the Supplemented Group than in the Unsupplemented Group, at the LPP. There was no significant difference between the two groups in the rate of maintenance of blood vitamin B12 at the EPP and LPP. The rate of maintenance of blood vita min $C$ did not differ significantly between the two 
groups at the EPP nor LPP, and remained very low in both groups.

\section{DISCUSSION}

TPN is associated with problems such as meta bolic acidosis and Wernicke encephalopathy, and other severe complications of micronutrient deficiency [4-10]. At present, it is therefore recommended that TPN should be accompanied by vitamin administration [11].

Regarding the need for vitamin administration during PPN, it has been believed that severe complications of vitamin deficiency are unlikely to occur since the amounts of calories and of carbohydrates administered are small during PPN and because the duration of PPN is usually short [12]. In this study, however, it was demonstrated that the blood vitamin B1 level about 3 days of PPN in about 79\% of the patients who received PPN throughout the periopera tive period without vitamin administration was below the lower limit ( $28 \mathrm{ng} / \mathrm{mL}$ ) of the criterion range proposed by the Committee for $\mathrm{V}$ itamin Laboratory Standards, Japan [17]. In about $71 \%$ of the same perioperative patients receiving PPN without vitamin administration, the blood vitamin B6 level was below the lower limit of the criterion range, and all of these patients also had blood vitamin $\mathrm{C}$ levels below the lower limit of the criterion range. Prior to the present study, there have been reported that about $20-40 \%$ of patients admitted to a critical care unit or patients who had undergone gastrointestinal surgery al ready had a very low blood vitamin B1 level before the start of PPN, and that their blood vitamin B1 level decreased further after PPN without vitamin adminis tration [18-19]. Shineha et al. [21] and Goseki and T suchiya [22] had al so reported that 40-60\% patients who had received gastrointestinal surgery had shown less than $28 \mathrm{mg} / \mathrm{ml}$ of blood vitamin B1 level after PPN. These findings suggested that PPN without vitamin administration in patients after gastrointestinal surgery could likely cause vitamin B1 deficiency.

It was reported that total body vitamin B1 depletion can occur within eighteen days [23], and deficiency in vitamin B1 occurs earlier than deficiency in any other vitamin if vitamin intake is stopped [24]. V itamin B1 deficiency presents very severe complications such as lactic acidosis and Wernicke encephalopathy, and the number of patients with these complications has not decreased in recent years $[4-10,25]$. Thus, among patients with various primary diseases, some are malnourished and poten- tially prone to vitamin deficiency.

From the point of view of prevention vitamin B1 deficiency, preparations for PPN supplemented with vitamin B1 have been developed recently. These preparations are expected to be very useful and safety. It has been reported that the use of these preparations during the period of fasting after gastrointestinal surgery enabled good maintenance of blood vitamin B1 level [21-22]. It has also been reported that in patients with shock or critically ill, loss of vitamin B increased, resulting in rapid onset of symptoms of vitamin B1 deficiency [26]. In patients after gastrointestinal surgery, fasting and stress increased metabolic rate, probably resulting in increased need for vitamins. Compared to previous studies [21-22], this study involved administration of large amounts of vitamin B1 over a short period of time. Although in the Supplemented Group, the blood vitamin B1 level was kept high, we cannot rule out the possibility that the vitamin B1 dosage was a little too much. In any event, it is noteworthy that discontinuation in supplement of vitamin after resumption of oral ingestion resulted in very rapid decrease in blood vitamin B1 level. Furthermore, in the Unsupplemented Group, the percentage of patients in whom the blood vitamin B1 level was kept above the lower limit of the criterion range at the postoperative fasted period was less than $25 \%$, while after resumption of oral ingestion this rate was still less than $40 \%$. The mean blood vitamin B1 was thus less than the lower limit of the criterion range in both the EPP and the LPP. These findings confirmed that vitamin B1 has a very short half-life in blood, and that its blood level decreases rapidly in the absence of vitamin B1 supplementation, and the blood vitamin B1 level does not recover rapidly even after resumption of peroral ingestion. It thus appears essential to administer vitamin B1 during postopera tive PPN.

In addition to vitamin B1, three other water-soluble vitamins (B6, B12, and C) were examined in this study.

The rate of maintenance of blood vitamin B6 level was significantly lower in the Unsupplemented Group than in the Supplemented Group, throughout the perioperative period. In the Supplemented Group, the blood vitamin B6 level began to increase soon after surgery, and returned to within the criterion range at the LPP. Although the method used in this study for administration of vitamin B6 may have involved an excessive administration of vitamin B6, no patient exhibited any adverse side-effect. Vitamin 
B6 functions as a coenzyme in the metabolism of glucose and protein. The requirement for vitamin B6 correspondingly appears to increase in the presence of stress. Although this study yielded no evidence directly linking blood vitamin levels with clinical outcome, it may be concluded that administration of vitamin B6 in the amounts needed by a given patient is essential for maintenance of blood vitamin B6 level within the criterion range.

The blood vitamin B12 level in 1 patient of the Unsupplemented Group ( $n=14)$ was lower than the criterion range at the postoperative fasting period. In the Supplemented Group ( $n=22), 16$ patients exhibited abnormally high blood vitamin B12 level above the upper limit of detection. This unexpected result might be explained by the following factors: (1) vitamin B12 has a long half-life in blood; and (2) the vitamin B12 dosages administered in this study were much higher than those recommended by the guidelines prepared by the A merican Medical A ssociation (A MA ) [27]. The method and dosages of administra tion of vitamin B12 used in this study appeared likely to show in excessive vitamin B12 blood levels. Further studies are needed to determine the optimal method for administration of vitamin B12 during PPN, however, vitamin B12 supplement dose not seem to be necessary at least for perioperative PPN treatment.

The number of patients in whom the blood vita min C level was within the criterion range before surgery was small in both the Supplemented Group ( 2 of 22 patients) and the Unsupplemented Group (5 of 18 patients). During the postoperative parenteral nutrition administration, the blood vitamin C level was below the criterion range in $95-100 \%$ of patients in both groups. Since vitamin C plays important roles in collagen cross linking, is required for wound healing, and reduces the postoperative oxidative stress, it can be considered an essential micronutrient during the perioperative period. Therefore, reduction in vita min $C$ intake may increase the risk to vitamin $C$ deficiency. In this study, the blood vitamin C level remained below the lower limit of the criterion range, even though once daily administration of vita min C was five times of recommended dose. It looks like huge amount of vitamin $C$ might be necessary during perioperative period. Further studies are necessary to determine the dosages and method for administration of vitamin $C$ for perioperative patients.

In this study, the blood vitamin levels were mea sured using the method recommended by the
Vitamin Standardization Committee [15]. It is still unclear to which extent blood vitamin levels reflect deficiency in vitamins in vivo and whether measurement of blood vitamin levels is clinically beneficial. To answer these questions in detail, it will be neces sary to investigate urinary elimination of vitamins and the cellular functions of vitamins. However, for water-soluble vitamins, the reserves of which are very low in vivo and whose half-lives in blood are very short, it seems necessary to keep blood levels within the criterion ranges proposed by the $\mathrm{V}$ itamin Standardization Committee.

Regarding administration of water-soluble vita mins to patients receiving PPN, the guidelines prepared by ASPEN and JSPEN basically recommend that they should be administered in amounts needed by each individual patient [11-12]. From this study, it was shown that the levels of water-soluble vitamins in blood during the brief period of postoperative PPN varied among vitamins. For safer and more effective PPN, the vitamin requirements and optimal methods for administration require further investigation for patients under various conditions. These results might be useful in the planning of such investiga tions. In conclusion, our studies suggested that administration of water-soluble vitamins during PPN was needed to avoid potential vitamin deficiencies after surgery and to prevent a potential onset of severe metabolic complications from any deficiencies.

\section{REFERENCES}

1. Dudrick SJ, Wilmore DW, Vars HM, and Rhoads JE. Long-term total parenteral nutrition with growth, development, and positive nitrogen balance. Surgery 1968; 64:134-142.

2. Quigley EM, Marsh MN, Shaffer JL, and Markin RS. Hepatobiliary complications of total parenteral nutrition. Gastroenterology 1993; 104:286-301.

3. Knapke CM, Owens JP, and Mirtallo JM. Management of glucose abnormalities in patients receiving total parenteral nutrition. Clin Pharm 1989; 8:136-144.

4. Velez RJ, Myers B, and Guber MS. Sever acute metabolic acidosis (acute beriberi): an avoidable complication of total parenteral nutrition. JPEN 1985; 9:216-219.

5. Thauvin-Robinet $\mathrm{C}$, Faivre $\mathrm{L}$, Barbier $\mathrm{ML}$, Chevret $\mathrm{L}$, Bourgeois J et al. Severe lactic acidosis and acute thiamin deficiency: A report of 11 neonates with unsupplemented total parenteral nutrition. J Inherit Metab Dis 2004; 27:700-704.

6. Kramer J, and Goodwin JA. Wernicke's encephal opathy. 
Complication of intravenous hyperalimentation. JAMA 1977; 238:2176- 2177.

7. Shikata E, Mizutani T, Kokubun $Y$, and Takasu T. 'Iatrogenic' Wernicke's Encephalopathy in Japan. Eur Neurol 2000; 44:156-161.

8. Nakasaki H, Ohta M, Soeda J, Makuuchi H, T suda M et al. Clinical and biochemical aspects of thiamine treatment for metabolic acidosis during total parenteral nutrition. Nutrition 1997; 13:110-117.

9. Kobayashi $\mathrm{Y}$, Kuroda H, Nanba H, Kobayashi I, and Sumita S. Severe Hypotension with Lactic Acidosis and Wernicke's Encephalopathy induced by TPN without Thiamine. J Intensive Care Med 2003; 27:709-713. (in Japanese)

10. Cho YP, Kim K, Han MS, Jang HJ, Kim JS et al. Severe lactic acidosis and thiamine deficiency during total parenteral nutrition-case report. Hepatogastroenterology 2004; 51:253-255.

11. A.S.P.E.N. Board of Directors and The Clinical Guidelines Task Force. Section VI: Normal Requirements A dults. Guidelines for the U se of Parenteral and Enteral Nutrition in Adult and Pediatric Patients. JPEN 2002; 26:22-24SA.

12. Japanese Society for Parenteral and Enteral Nutrition : Practical Guidelines for Parenteral and Enteral Nutrition, Herusu Shuppan, Tokyo, pp 9-11, 1998. (in Japanese)

13. I wase K, Higaki J, Y oon HE, Mikata S, Tanaka Y et al. A Case of Metabolic A cidosis due to V itamin B1 Deficiency Following Intravenous Infusion for Dehydration in a Gastrectomized Patient. J Jpn Surg A ssoc 2000; 61:23472351. (in Japanese)

14. Iwase K, Houki M, Ito T, Mizuno H, Mizushima T et al. A Case of Wernicke's Encephalopathy during Peripheral Parenteral Nutrition in a Gastrectomized Patient. J Jpn College of Surgeons 2005; 30:712-715. (in Japanese)

15. Mino M. Standard Method on V itamin Determination for Clinical Samples, as recommended by the Vitamin Society of Japan. Vitamin 2000; 74:501-515. (in Japanese)

16. Japanese Gastric Cancer A ssociation: Japanese classifica tion of gastric carcinoma -2nd English edition-. Gastric Cancer 1998; 1:10-24.

17. Itokawa Y, Hashizume N, A sano M, Igarashi O, Mino M et al. Proposed standard for human blood vitamin B1 value using HPLC. The Committee for Vitamin Laboratory Standards, Japan. Biofactors 1999; 10:295299.

18. Ihara $\mathrm{H}$, and Hashizume $\mathrm{N}$. Evidence-based medicine (EBM) for assessment of vitamin deficiency. Current review of clinical pathology 2003; 127:24-30. (in Japanese)

19. Cruickshank AM, Telfer AB, and Shenkin A. Thiamine deficiency in the critically ill. Intensive Care Med 1988; 14:384-387.

20. Fukushima R, Shiratori M, Y ago H, Ogihara T, Ogawa $F$ et al. Should vitamin B1 be added to peripheral parenteral nutrition after major gastrointestinal surgery? Determination of perioperative vitamin B1 status. Jpn J Surg metabolism and nutrition 2003; 37:23-31. (in Japanese)

21. Shineha R, T suchiya T, Y amasaki S, T anihata E, Goseki N et al. Multicenter comparative phase III clinical study of A FV-03, an intravenous amino acid and glucose solutiion with electrolytes and vitamin B1, in patients after gas trointestinal surgery. J New Rem \& Clin 2006; 55:305338. (in Japanese)

22. Goseki N, and Tsuchiya T. Clinical significance of coadministration of vitamin B1 and its dosage in peripheral parenteral nutrition. J New Rem \& Clin 2006; 55:339350. (in Japanese)

23. MacLean LD, Rhode BM, and Shizgal HM. Nutrition following gastric operations for morbid obesity. Ann Surg 1983; 198:347- 355.

24. Bates CJ. Chapter 17 Thiamin (Kimura M) . Present knowledge in nutrition. Bowman BA, Russel RM (supervision of Kimura $S$ and Kobayashi $S$ ), 8th edn, Kenpakusya, Tokyo , pp189-195, 2002.

25. Kitamura K, Y amaguchi T, Tanaka H, Hashimoto S, Y ang $M$ et al. TPN-induced fulminant beriberi: a report on our experience and a review of the literature. Surg Today 1996; 26:769- 776.

26. McConachie I, and Haskew A. Thiamine status after major trauma. Intensive Care Med 1988; 14:628-631.

27. A merican Medical Association Department of Foods and Nutrition, 1975 : Multivitamin Preparations for Parenteral Uses, A Statement by the Nutrition Advisory Group. JPEN 1979; 3:258- 262. 